The following article is the final version submitted to IEEE after peer review; hosted by PTB; DOI: 10.1109/AMPS50177.2021.9586012. It is provided for personal use only.

\title{
Traceable calibration system for non-conventional current sensors with analogue or digital output
}

\author{
Y. Chen, E. Mohns, M. Seckelmann and S.de Rose
}

Acknowledgement: The presented work is used in the project 17IND06 (FutureGrid II) which have received funding from the EMPIR programme co-financed by the Participating States and from the European Union's Horizon 2020 research and innovation programme.

(C) 2018 IEEE. This is the author's version of an article that has been published by IEEE.

Personal use of this material is permitted. Permission from IEEE must be obtained for all other uses, in any current or future media, including reprinting/republishing this material for advertising or promotional purposes, creating new collective works, for resale or redistribution to servers or lists, or reuse of any copyrighted component of this work in other works.

Full Citation of the original article published be IEEE:

Y. Chen, E. Mohns, M. Seckelmann and S. de Rose, "Traceable calibration system for non-conventional current sensors with analogue or digital output," 2021 IEEE 11th International Workshop on Applied Measurements for Power Systems (AMPS), 2021, pp. 1-6, doi: 10.1109/AMPS50177.2021.9586012.

Available at: https://ieeexplore.ieee.org/document/9586012 


\section{Traceable calibration system for non-conventional current sensors with analogue or digital output}

\author{
Yeying Chen \\ dept. 2.3 - Electrical Energy Measuring \\ Techniques \\ Physikalisch-Technische Bundesanstalt \\ (PTB) \\ Braunschweig, Germany \\ yeying.chen@ptb.de
}

Soeren de Rose

dept. 2.3 - Electrical Energy Measuring Techniques

Physikalisch-Technische Bundesanstalt (PTB)

Braunschweig, Germany

soeren.de-rose@ptb.de

\author{
Enrico Mohns \\ dept. 2.3 - Electrical Energy Measuring \\ Techniques \\ Physikalisch-Technische Bundesanstalt \\ (PTB) \\ Braunschweig, Germany \\ enrico.mohns@ptb.de
}

\author{
Michael Seckelmann \\ dept. 2.3 - Electrical Energy Measuring \\ Techniques \\ Physikalisch-Technische Bundesanstalt \\ (PTB) \\ Braunschweig, Germany \\ Michael.Seckelmann@ptb.de
}

\begin{abstract}
This paper describes the setup of a traceable calibration system for conventional or non-conventional current sensors even with digital output. The system is lately developed at PTB within the frame of the European project "FUTUREGRID II". Details of the system components are presented. The absolute phase errors of the two-channel generator related to the pulse per second time reference of the global positioning system can be configured to almost zero. The accuracies of the reference current transformers are within $\pm 10 \mu \mathrm{A} / \mathrm{A}$ and $\mu \mathrm{rad}$ at power frequency. The sampled value receiver box is firstly validated for the sample rate of $4 \mathrm{kHz}$ according to the IEC standard 61869-9 [1].
\end{abstract}

Keywords - digital, current transformer, merging unit, standalone merging unit, traceability, calibration, sampled values

\section{INTRODUCTION}

Digitalization is the core of the future electrical power grid. The instrumentations in power grid substations are moving from an "iron and copper" state to a more multifaceted and intelligent grid, where the stability under increasingly complex and challenging conditions is required for real-time capable control and monitoring systems. The European project "FutureGrid II" is committed to developing new techniques and calibration services with special emphasis on accurate timing for the measurement equipment with digital input and output according to IEC 61850-9-2 protocol [2]. Such services are not yet widely available in national metrology institutes worldwide [3]. In general, instrumentation in electricity transmission and distribution systems are responsible for monitoring from general metering to protection and grid diagnostics. Intensive research and development of new technologies for power generation, e.g., renewable technologies such as solar, wind, hydro, geothermal, and ocean-thermal conversion plants, accelerate the supervision of Power Quality (PQ) and Phasor Measurement Units (PMUs) [4]. In this context, the concept of the digital instrumentation, i.e. instrument transformers (ITs) associated with merging unit (MU) or stand-alone merging unit (SAMU) [5] emerges. The evolution for an electrical power grid with digital instrumentation is in full swing and poses a wide range of challenges for all stakeholders. Investments in innovative technologies today create future-proof power grids characterized by reliability, efficiency, and sustainability [6], [7].
The input of the FutureGrid II project contributes to the long-term research and innovation activities and the European Union's energy transition and the modernisation of the electricity grid. One of the objectives of FutureGrid II is to develop new test systems for the dynamic characterisation of the ITs for PQ measurements and to exploit the necessary metrological infrastructure and standards. The calibration systems support dynamic testing of emerging nonconventional transformers, i.e. analogue and digital ITs, including tests with typical waveforms associated with PQ and synchronised phasor measurements. In this paper, the setup of a calibration system for rated currents up to $2 \mathrm{kA}$ is introduced for any kind of analogue current sensors (conventional or nonconventional current transformers) or current transformers (CTs) with digital output, which includes the associated MU/SAMU.

\section{SETUP OF THE CALIBRATION SYSTEM FOR THE CTS}

The setup of the calibration system for the current transformers is shown in Fig. 1.

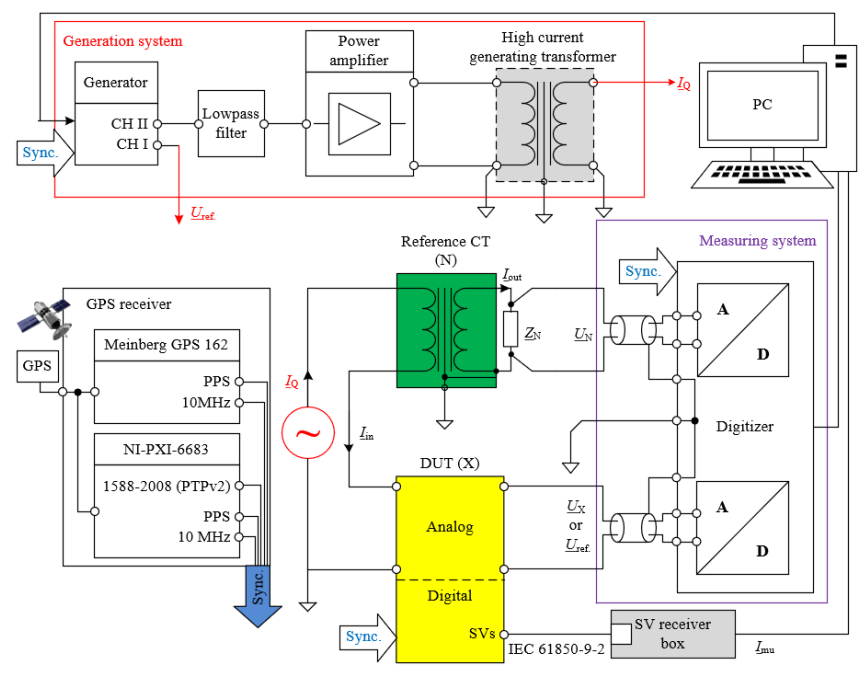

Fig. 1. The setup of a calibration system for current transformer calibrations

The calibration system is appropriate for any kind of analogue current sensors (conventional or non-conventional CTs) or CTs with digital output, which include the associated MU/SAMU. The calibration system is mainly made up of a 
high current generation system (marked as red block), a set of analogue reference CTs (marked as green block) with associated precision resistors [8] and a precision 2-channel measuring system (marked as purple block) [9], [10]. The synchronization signals (marked as "Sync." in blue) and the SV receiver box (shown as a grey block) are necessary when the output of the device under test (DUT) is a sampled value (SV) data stream.

The high current generation system consists of a programmable two-channel arbitrary waveform generator, a lowpass filter, a transconductance power amplifier and the high current generating transformers. Firstly, the arbitrary waveform generator, e.g., the Agilent 33500B Series with resolution of $16 \mathrm{Bit}$, generates different test waveforms, which reproduce PQ phenomena. The lowpass filter is to used limit the slew rate and the bandwidth of the generated signal with adjustable amplitudes. The generated signal is in turn amplified by the transconductance power amplifier (up to $270 \mathrm{~V}_{\text {rms }} / 70 \mathrm{~A}_{\mathrm{rms}}$, DC to $15 \mathrm{kHz}$ ) and by the high current generation transformers with several rated current ranges from $5 \mathrm{~A}$ to $1 \mathrm{kA}$ rated. The laboratory generation capabilities are tested with frequencies ranging from $50 \mathrm{~Hz}$ to $5 \mathrm{kHz}$. Sinusoidal waveforms up to $2 \mathrm{kA}$, dual-tone or multi-tone waveforms up to $2 \mathrm{kA}$ and amplitude modulated waveforms are generated. The limitation at frequencies above $500 \mathrm{~Hz}$ is caused by the internal stray inductance of the high current generation transformer.

The synchronization plays a significant role for the phase measurement processes of the SV-based devices and the digital signal processing between different devices within the substation. The synchronization signals can be obtained by two laboratory global positioning system (GPS) receivers: the "Meinberg GPS 162" and the "NI-PXI-6683". The synchronization signals are regarded as the time references for the calibration systems and are transmitted as $10 \mathrm{MHz}$, pulse per second (PPS) and IEEE 1588-2008 (PTPv2) protocol. Besides, the White Rabbit LENs [11], which are completely Ethernet-based networks, are used for the time data transmission and synchronization in the laboratory with subnanosecond precision.

The deduction of the calibration error for the current transformers is split into two parts. For the conventional CT calibrations, the DUT is regarded as an analogue CT. The complex voltage ratio $\underline{\Gamma}_{\mathrm{XN}}$ of the measuring system, $\underline{\Gamma}_{\mathrm{XN}}=\underline{U}_{\mathrm{X}} / \underline{U}_{\mathrm{N}}$, is measured by a Digitizer and two calibrated shunts: $\underline{Z}_{\mathrm{N}}$ and $\underline{Z}_{\mathrm{X}}$. Using the ratio $\underline{F}_{\mathrm{IU}, \mathrm{N}}$ of the reference current-to-voltage $\left(\mathrm{C}\right.$-to-V) transformer, $\underline{F}_{\mathrm{IU}, \mathrm{N}}=\underline{U}_{\mathrm{N}} / \underline{I}_{\text {in }}$ $=\underline{U}_{\mathrm{N}} / \underline{I}_{\text {out }} \cdot \underline{I}_{\text {out }} / \underline{I}_{\text {in }}=\underline{Z}_{\mathrm{N}} \cdot \underline{F}_{\mathrm{I}, \mathrm{N}}$, and the ratio $\underline{F}_{\mathrm{IU}, \mathrm{X}}$ of the DUT C-to-V transformer, $\quad \underline{F}_{\mathrm{IU}, \mathrm{X}}=\underline{U}_{\mathrm{X}} / \underline{I}_{\text {in }}=\underline{U}_{\mathrm{X}} / \underline{I}_{\text {out }} \cdot \underline{I}_{\text {out }} / \underline{I}_{\mathrm{P}}$ $=\underline{Z}_{\mathrm{X}} \cdot \underline{F}_{\mathrm{I}, \mathrm{X}}$, the current ratio $\underline{F}_{\mathrm{I}, \mathrm{X}}$ of the DUT CT can be calculated as follows:

$$
\underline{F}_{\mathrm{I}, \mathrm{X}}=\underline{\Gamma}_{\mathrm{XN}} \cdot \underline{F}_{\mathrm{I}, \mathrm{N}} \cdot \underline{Z}_{\mathrm{N}} / \underline{Z}_{\mathrm{X}}
$$

Besides, the $\underline{F}_{\mathrm{I}, \mathrm{X}}$ for a CT (DUT) can be defined as

$$
\underline{F}_{\mathrm{I}, \mathrm{X}}=\underline{I}_{\mathrm{S}} / \underline{I}_{\mathrm{P}}=1 / K_{\mathrm{n}} \cdot\left(1+\varepsilon_{\mathrm{i}}\right) \cdot \mathrm{e}^{\mathrm{j} \cdot \delta \mathrm{i}}
$$

where $K_{\mathrm{n}}$ is the transformer ratio of the rated primary and secondary currents $K_{\mathrm{n}}=I_{\mathrm{P}, \mathrm{r}} / I_{\mathrm{S}, \mathrm{r}}, \varepsilon_{\mathrm{i}}$ and $\delta_{\mathrm{i}}$ represent the ratio error and the phase error of the CT. By combining (1) and (2), the errors of the analogue CT are deduced.
For a digital instrument transformer, the ratio $\underline{F}_{\mathrm{I}, \mathrm{X}}$ can be expressed by $\underline{I}_{\mathrm{mu}} / \underline{I}_{\mathrm{in}}$ (or equivalently $\underline{U}_{\mathrm{mu}} / \underline{U}_{\mathrm{in}}$ ). $\underline{I}_{\mathrm{mu}}$ represents the complex fundamental rms current that is calculated from the Discrete Fourier Transform (DFT) of the SVs. $\underline{I}_{\text {in }}$ refers to the measured primary current by means of the reference CT $\underline{F}_{\mathrm{I}, \mathrm{N}}$, the highly accurate shunt $\underline{Z}_{\mathrm{N}}$ and the measured voltage $\underline{U}_{\mathrm{N}}$ of the digitiser. The measured complex primary current is determined according to $\underline{I}_{\text {in }}=\underline{U}_{\mathrm{N}} /\left(\underline{Z}_{\mathrm{N}} \cdot \underline{F}_{\mathrm{I}, \mathrm{N}}\right)$. In order to relate the two measurements, a sinusoidal signal $\underline{U}_{\text {ref }}$ is generated as the phase reference by the waveform generator, programmed without any phase offset $\left(\arg \left\{\underline{U}_{\text {ref }}\right\}=0^{\circ}\right)$ at channel CH I. This phase reference, which is synchronised to the GPS PPS signal, is connected the Digitiser channel $\underline{U}_{\mathrm{X}}\left(\underline{U}_{\mathrm{X}}=\underline{U}_{\mathrm{ref}}\right)$. The complex voltage ratio $\underline{\Gamma}_{\mathrm{XN}}$ is measured by a Digitizer $\underline{\Gamma} \underline{\mathrm{XN}}_{\mathrm{N}}=\underline{U}_{\mathrm{X}} / \underline{U}_{\mathrm{N}}=\underline{U}_{\mathrm{ref}} /\left(\underline{I}_{\mathrm{in}} \cdot \underline{Z}_{\mathrm{N}} \cdot \underline{F}_{\mathrm{I}, \mathrm{N}}\right)$. The ratio error $\varepsilon_{\mathrm{i}}$ of the digital DUT can be calculated as follows:

$$
\varepsilon_{\mathrm{i}}=I_{\mathrm{mu}} / \underline{I}_{\mathrm{in}}-1
$$

where the rms current $I_{\text {in }}$ is determined by $I_{\text {in }}=U_{\mathrm{N}} /\left(Z_{\mathrm{N}} \cdot F_{\mathrm{I}, \mathrm{N}}\right)$. The phase error $\delta_{\mathrm{i}}$ of the digital DUT can be calculated as

$$
\delta_{\mathrm{i}}=\arg \left\{I_{\mathrm{mu}}\right\}-\arg \left\{\underline{I}_{\mathrm{in}}\right\}
$$

where the absolute phase of the complex current $\arg \left\{\underline{I}_{\text {in }}\right\}$ is determined by $\arg \left\{\underline{I}_{\text {in }}\right\}=\arg \left\{\underline{U}_{\text {ref }}\right\}-\left(\arg \left\{\underline{X X N}_{\mathrm{XN}}\right\}+\arg \left\{\underline{Z}_{\mathrm{N}}\right\}\right.$ $\left.+\arg \left\{\underline{F}_{\mathrm{I}, \mathrm{N}}\right\}\right)$.

\section{COMPONENTS OF THE CALIBRATION SYSTEM}

The detailed components for the current transformer calibration system, shown in Fig. 1, deal with a programmable two-channel generator for the generation system, a reference current-to-voltage (C-to-V) transformer set as analogue reference CTs and an $\mathrm{SV}$ receiver box for the CTs with digital output.

\section{A. Program of the two-channel generator}

The LabVIEW program is designed to control and extend the waveform generation of the two-channel generator with three main functions: hardware communication, generation setups and the data stream generation. The flow chart of software is shown in Fig. 2.

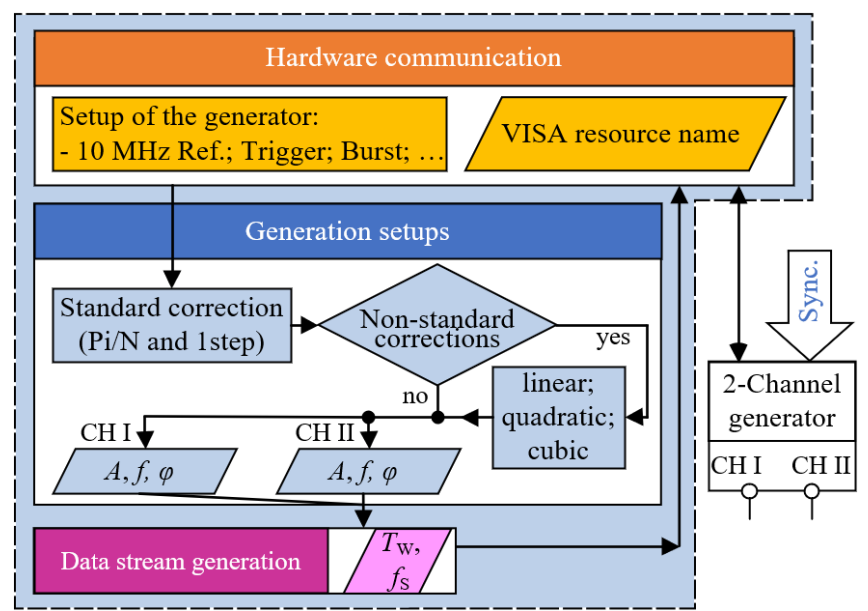

Fig. 2. Flow chart of the Agilent generator LabView-based software platform

The hardware communication (shown as orange blocks) must be correctly set before the signals are sent to the generator. The generation setups (shown as blue blocks) contain the general settings of the signal parameters $(A, f, \varphi)$, and the phase corrections. Based on the sample rate $f_{\mathrm{S}}$ and the 
time duration $T_{\mathrm{W}}$ (shown in the purple block), the arbitrary waveforms are calculated as a data set. Finally, this data set is sent to the generator.

The phase corrections are used for the alignment of the arbitrary waveforms to the reference PPS signal. The phase corrections are differentiated into standard corrections and optionally usable, small non-standard corrections for compensating the generator-internal phase shifts. To avoid the time delay between the reference PPS and the generated signals, a numerical method is designed. The "ideal" mathematical time-quantized voltage waveform is expressed as

$$
u_{\mathrm{DAC}}(v)=\sqrt{ } 2 \cdot U_{\mathrm{rms}} \cdot \sin \left(B \cdot v+\varphi_{0}+\varphi_{\text {step }}+x+y\right)
$$

where $0 \leqslant v \leqslant T_{\mathrm{w}} \cdot f_{\mathrm{s}}-1, B=2 \pi / N, x=\pi / N$ (" $\mathrm{Pi} / \mathrm{N} "$ phase correction [12], [13]), $\varphi_{\text {step }}=2 \pi / N$ ("1-step" phase correction [12], [13]), $N=f_{\mathrm{s}} / f$ (samples per period - SpP) and $y$ refers to the user-defined non-standard phase corrections.

\section{B. The reference $C$-to- $V$ transformer}

The reference $\mathrm{C}$-to- $\mathrm{V}$ transformer consists of a current transformer and an associated precise measuring resistor. The rated primary currents of the $\mathrm{C}$-to- $\mathrm{V}$ transformer set ranges from $8.3 \mathrm{~A}$ to $1500 \mathrm{~A}$. Four commercial zero-flux CTs with rated currents of $50 \mathrm{~A}$ (CT50), $200 \mathrm{~A}$ (CT200 [8]), $600 \mathrm{~A}$ (CT600) and $2 \mathrm{kA}$ (CT1500) were used to establish the reference CTs . To convert the different secondary currents of these CTs into a $1 \mathrm{~V}$ output voltage, a resistor box was built, containing six precision resistors from $1 \Omega$ to $20 \Omega$.

As an example, the symmetrical winding configuration of the added primary of the CT1500 is illustrated in detail in Fig. 3 (a) from a vertical view. The connection scheme for the different primary windings $N_{\mathrm{p}}=1$ to 4 of the CT1500 is shown in Fig. 3 (b).

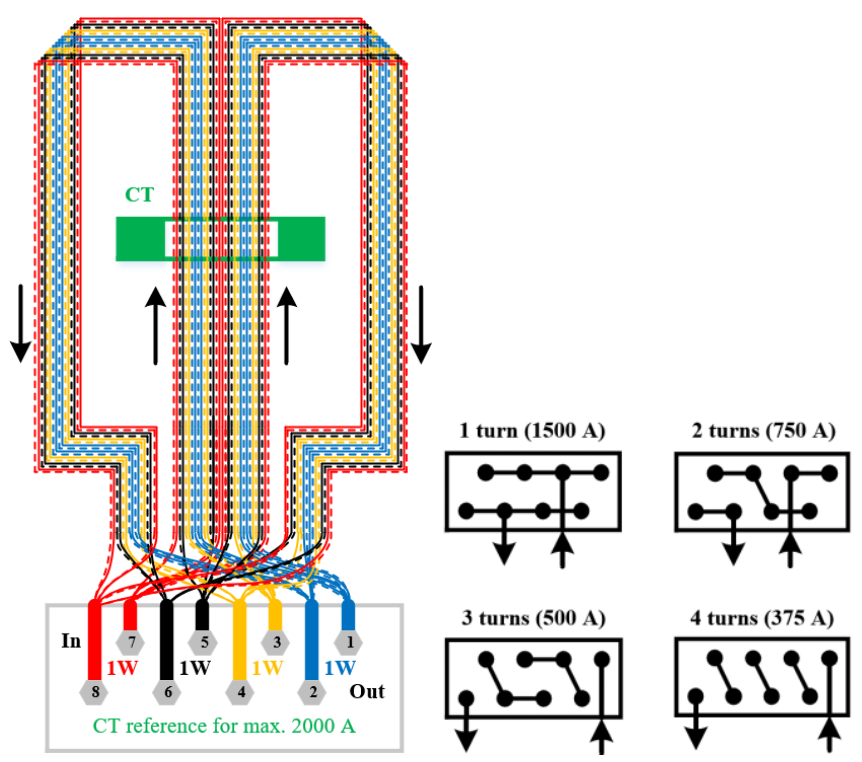

Fig. 3. Configuration design (left) and connection setups (right) of CT1500

The usability and expected metrological characteristics of the window type zero-flux transformer were improved by implementing several symmetrical primary windings to the CT.

The resistor box consists of two power supplies, two separate channels and six precision resistors. The power supply with $\pm 15 \mathrm{~V}$ DC voltage operates the CTs. The integration of two channels into one box allows a simple and monolithic calibration facilities for the reference CT as against the DUT. The nominal values of the resistors were selected from $1 \Omega$ to $20 \Omega$. The detailed designs of one measuring resistor are schematically shown in Fig. 4 from various views.

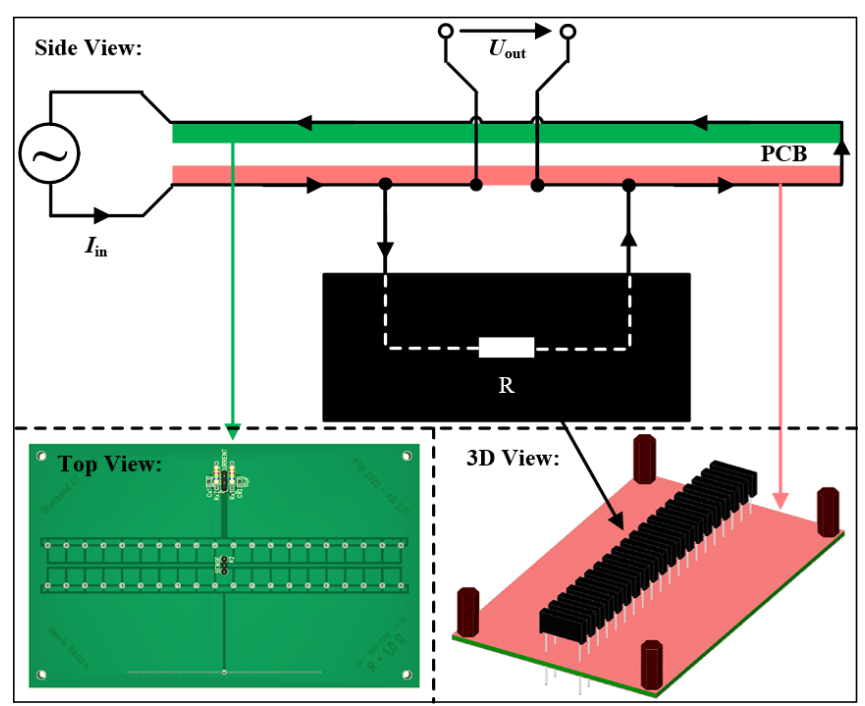

Fig. 4. Designs of the measuring resistor from various views (without a heatsink)

Each measuring resistor $R_{\mathrm{m}}$ is designed to be made up of numbers of resistors $R$ in parallel, an associated printed circuit board (PCB) and a large heatsink. Each single resistor $R$ was selected with a power rating of $1.5 \mathrm{~W}$. The construction of the resistors $R$ in parallel for one $R_{\mathrm{m}}$ value, e.g., twenty $20 \Omega$ resistors in parallel for a $1 \Omega$ measuring resistor, benefits to reduce the power loss inside the resistor. The PCB is designed to minimize the ac errors $\left(\varepsilon_{\mathrm{m}}\right.$ and $\left.\delta_{\mathrm{m}}\right)$ of the measuring resistor by minimizing the effective magnetic field for the resistors. The heatsink is developed later around the resistors.

\section{SV receiver box}

The self-designed SV receiver is developed to connect SVbased devices with the LabView-based software platform in a computer with integrated analysis of the SV.

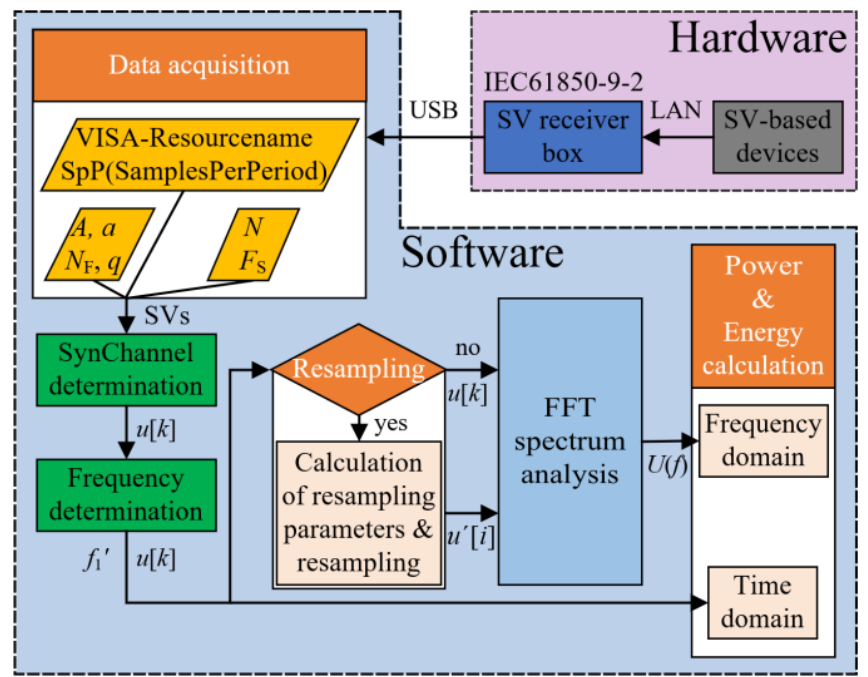

Fig. 5. Flow chart of the SV receiver box LabView-based software platform with hardware connections

To avoid damaging of the sampled values, the SV receiver box offers PC time buffer for a seamless, quasi-real-time digital data processing. In praxis, the SVs, which satisfied 
with the standard IEC61869-9, are sent from the SV-based devices, e.g., SAMU/MU, through an Ethernet port. The SV receiver transmits the SVs to the computer through a USB port according to IEC61850-9-2 communication protocol.

The flow chart of the LabView-based software platform, shown in Fig. 5, illustrates the detailed processes according to different function modules. Firstly, the VISA-Resourcename and parameters $\left(\mathrm{SpP} ; A, a, N_{\mathrm{F}}\right.$ and $q$ : the resampling parameters; $N, F_{\mathrm{s}}$ : the sampling parameters) are required for the data acquisition. After the transfer of the data (SVs), the channel for the synchronisation (SynChnnel) is selected and the frequency of the signal is determined. The resampling processing [14] can be chosen when the detected signal frequency is not synchronised to the given sampling parameters. The FFT spectrum analysis is supposed to determine rms values and phase angles of the voltages and currents of the SV in the frequency domain. Based on these values, the required current $I_{\mathrm{mu}}$ and its phase $\arg \left\{I_{\mathrm{mu}}\right\}$ can be extracted for calculating the current error and phase displacement - see (3) and (4) - of the SV-based device.

\section{VALIDATIONS OF THE CALIBRATION SYSTEM}

The validations of the CT calibration system, corresponding to the described components in Section III, are divided into three parts: results of the programmable twochannel generator, characterisation of the reference C-to-V transformer set and validations of the SVs.

\section{A. Calibration results of the programmable two-channel generator}

The calibrations with the standard phase corrections (Pi / N \& 1 step phase corrections) for the programmable twochannel generator were divided into two aspects: the characterization of the absolute phase shift between the generated signals of the generator and the GPS PPS time reference and the characterization of the relative phase shift between the two generated signals of the two channels. The absolute phase error $\Delta \varphi_{\text {abs }}$ was determined from the time difference $\Delta t_{\mathrm{abs}}$ between the zero gear of the generated phase reference signal $t_{\mathrm{CHI}}$ and the positive edge of the PPS clock

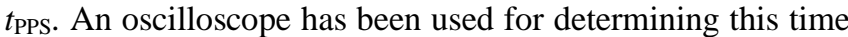
difference $\Delta t_{\mathrm{abs}}=t_{\mathrm{CHI}}-t_{\mathrm{PPS}}$. The relative phase error $\Delta \varphi_{\mathrm{rel}}$ between the two channels was measured by the precision 2channel measuring system [15].

The investigated parameters for the calibration measurements are classified into two groups for each characterization aspect: the generation parameters of the SV data stream $\left(f_{\mathrm{s}}\right.$, i.e., the $\mathrm{SpS}$ and $\left.T_{\mathrm{W}}\right)$ and the general settings of the signal parameters $\left(f_{0}, A_{0}, \varphi_{0}\right.$ and $f_{\mathrm{h}}, A_{\mathrm{h}}, \varphi_{\mathrm{h}}$ for a dual-tone signal with the $h^{\text {th }}$ overtone as well as $f, A, \varphi$ for a sinusoidal or square signal).

a) Absolute phase errors $\Delta \varphi_{\mathrm{abs}}$ : For example, a measurement result for deriving the absolute phase errors $\Delta \varphi_{\text {abs }}$ is shown in Fig. 6, which contains many modified details from the oscilloscope screen shot. The used parameter values were regarded as default values for the general measurement. Besides, since the resolution of the oscilloscope is in nanosecond range, the oscilloscope measurement almost overlaps the generated signals from the two-channel generator.

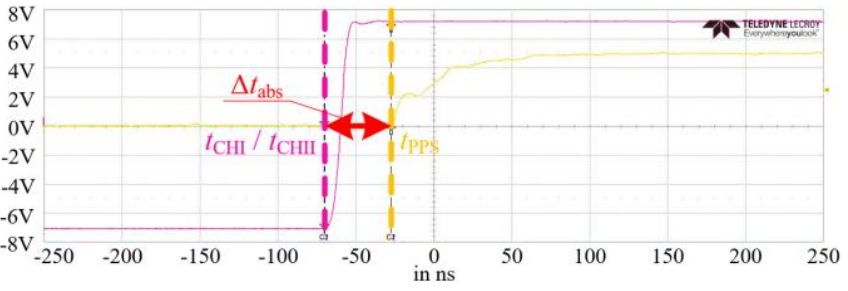

Fig. 6. The time difference between the zero gear of the generated signal (the pink curve) and the positive edge of the PPS clock (the yellow curve) in the oscilloscope. (square wave - general settings: $f=50 \mathrm{~Hz}, U=5 \mathrm{~V}$; the generation parameters: $f_{\mathrm{s}}=100 \mathrm{kHz}, T_{\mathrm{W}}=1 \mathrm{~s}$; with $\mathrm{Pi} / N$ \& 1 step phase corrections)

For the measurement shown in Fig. 6, the time difference $\Delta t_{\text {abs }}$ (marked as red in Fig. 6) is obtained by the generated signal of one channel (shown as a pink curve) and the reference PPS clock (shown as a yellow curve). To avoid the reading error due to the reflection of the electric signals in the coaxial cable, the times $t_{\mathrm{CHI}} / t_{\mathrm{CHII}}$ and $t_{\mathrm{PPS}}$ were all directly determined at the beginning of the rising edge of the square wave signals or at the beginning of the positive edge for the sinusoidal signals. Finally, the absolute phase errors were calculated as $\Delta \varphi_{\mathrm{abs}}=2 \pi f \cdot \Delta t_{\mathrm{abs}}$.

Preliminary investigations on the absolute phase errors $\Delta \varphi_{\text {abs }}$ of the generated signals from the two-channel generator showed that the amplitude linearity $\left(A_{0}\right)$, the phase linearity $\left(\varphi_{0}\right)$ of the generated signals and the time window for the SV generation of the generator $\left(T_{\mathrm{W}}=1 \mathrm{~s}\right.$ or $\left.10 \mathrm{~s}\right)$ had no significant influence on $\Delta \varphi_{\text {abs. }}$ Moreover, the absolute phase errors of a sinusoidal signal had almost the same errors as its corresponding dual-tone signals $\left(A_{\mathrm{h}}=10 \% A_{0}, h\right.$ up to 51$)$. By varying fundamental frequency of the signals at $50 \mathrm{~Hz}, 60 \mathrm{~Hz}$ and $100 \mathrm{~Hz}$, the absolute phase errors $\left(\Delta \varphi_{\text {abs_ } 50 \mathrm{~Hz}}=-7.7 \mu \mathrm{rad}\right.$, $\left.\Delta \varphi_{\text {abs } 60 \mathrm{~Hz}}=-10.6 \mu \mathrm{rad}, \Delta \varphi_{\text {abs } \_100 \mathrm{~Hz}}=-15.6 \mu \mathrm{rad}\right)$ had small a difference of below $10 \mu \mathrm{rad}$ with $\mathrm{SpS}=100,000$.

In order to have a better recognition of the results from the oscilloscope's display, the square wave signals were generated for the measurements of SV generation with various SpS. To avoid the surrounding disturbance of the signals at power frequency, the signal frequency was set as $52 \mathrm{~Hz}$. Moreover, different measurement setup, e.g., various cable length for the connections, leads to a small drift for the absolute phase errors in $\mu$ rad range. The results regarding the different $\mathrm{SpS}$ ranging from 5,000 to 500,000 with a certain measurement setup are shown in TABLE I.

TABLE I. ABSOLUTE PHASE ERRORS OF THE GENERATED SIGNALS FROM THE TWO-CHANNEL GENERATOR REGARDING THE SPP WITH PI / N \& 1 STEP PHASE CORRECTIONS

\begin{tabular}{|c|c|c|c|c|c|}
\hline $\mathbf{S p S}$ & $\mathbf{S p P}$ & $\begin{array}{c}\Delta t_{\text {abs }}(\mathbf{C H} \\
\text { I - PPS }) \\
\text { in } \boldsymbol{\mu S}\end{array}$ & $\begin{array}{c}\Delta \varphi_{\text {abs }}(\mathbf{C H} \\
\text { I - PPS }) \\
\text { in } \boldsymbol{\mu r a d}\end{array}$ & $\begin{array}{c}\Delta t_{\text {abs }}(\mathbf{C H} \\
\text { II-PPS }) \\
\text { in } \boldsymbol{\mu S}\end{array}$ & $\begin{array}{c}\Delta \varphi_{\text {abs }}(\mathbf{C H} \\
\text { II - PPS }) \\
\text { in } \boldsymbol{\mu r a d}\end{array}$ \\
\hline $\mathbf{5 0 0 0}$ & $\mathbf{9 6 . 2}$ & -5.65 & -1845.88 & -5.65 & -1846.54 \\
\hline $\mathbf{1 0 0 0 0}$ & $\mathbf{1 9 2 . 3}$ & -2.70 & -883.56 & -2.71 & -884.05 \\
\hline $\mathbf{2 5 0 0 0}$ & $\mathbf{4 8 0 . 8}$ & -0.94 & -306.21 & -0.94 & -306.26 \\
\hline $\mathbf{5 0 0 0 0}$ & $\mathbf{9 6 1 . 5}$ & -0.35 & -114.21 & -0.35 & -113.99 \\
\hline $\mathbf{7 5 0 0 0}$ & $\mathbf{1 4 4 2 . 3}$ & -0.16 & -51.15 & -0.16 & -50.84 \\
\hline $\mathbf{1 0 0 0 0 0}$ & $\mathbf{1 9 2 3 . 1}$ & -0.06 & -18.93 & -0.06 & -18.70 \\
\hline $\mathbf{1 1 6 0 0 0}$ & $\mathbf{2 2 3 0 . 8}$ & 0.00 & -1.01 & -0.02 & -4.95 \\
\hline $\mathbf{1 5 0 0 0 0}$ & $\mathbf{2 8 8 4 . 6}$ & 0.06 & 18.41 & 0.04 & 14.38 \\
\hline $\mathbf{2 5 0 0 0 0}$ & $\mathbf{4 8 0 7 . 7}$ & 0.12 & 38.50 & 0.12 & 38.99 \\
\hline $\mathbf{5 0 0 0 0 0}$ & $\mathbf{9 6 1 5 . 4}$ & 0.12 & 38.40 & 0.12 & 38.77 \\
\hline
\end{tabular}

The generated signals are square wave signals with parameters of $U=5 \mathrm{~V}, f_{0}=52 \mathrm{~Hz}$

The results from TABLE I demonstrate that the absolute phase errors of the two channels of the generator were almost 
identical with each other for a certain $\mathrm{SpS}$. With increasing of the $\mathrm{SpS}$, the absolute phase errors changed from around $1846 \mu \mathrm{rad}$ with $\mathrm{SpS}=5,000$ to around $+38 \mu \mathrm{rad}$ with $\mathrm{SpS}=500,000$. After several attempts, the absolute phase errors approached to zero with $\mathrm{SpS}=116,000$. For the calibrations of a CT with a digital output, the absolute phase errors in TABLE I can be used directly as the phase corrections to the PPS signal, $\arg \left\{\underline{U}_{\mathrm{PPS}}\right\}$, for the reference phase, $\arg \left\{\underline{U}_{\text {ref }}\right\}$, in (4).

b) Relative phase errors $\Delta \varphi_{\mathrm{rel}}$ : The calibration measurements were executed by five aspects: the amplitude linearity (from $2 \% \cdot A_{0}$ to $100 \% \cdot A_{0}$ ) of the investigated signal, the phase linearity ( $\varphi_{0}$ from 0 degree to 180 degree) of the investigated signal, various generator clock rate $\left(f_{\mathrm{s}}\right.$ from $10 \mathrm{kHz}$ to $500 \mathrm{kHz}$ ) for the SV generation of the generator, various time window ( $T_{\mathrm{W}}$ from $1 \mathrm{~s}$ to $10 \mathrm{~s}$ ) for the $\mathrm{SV}$ generation of the generator and the influence by a dual-tone signal with an odd harmonics (up the $49^{\text {th }}$ order). One generated signal of the generator was set as an invariant reference signal $\left(A_{0}=5 \mathrm{~V}, f_{0}=52 \mathrm{~Hz}\right)$, which was connected to the reference channel of the measuring system. The parameters: $f_{\mathrm{s}}\left(\right.$ default: $\left.f_{\mathrm{s}}=100 \mathrm{kHz}\right), T_{\mathrm{W}}\left(\right.$ default: $\left.T_{\mathrm{W}}=1 \mathrm{~s}\right)$ and the option for $\mathrm{Pi} / N \& 1$ step phase corrections were programmed to be varied contemporaneously for both channels of the generator. As a result, the relative phase errors between the generated signals from two-channel generator stay below 10 urad for the complete investigations.

\section{B. Characterisation of the reference $C$-to-V transformer}

The final accomplished set of reference current transformers and the associated precise measuring resistor box are presented in Fig. 7. The reference CTs, shown in Fig. 7 (above) from link to right, are respectively the CT50, CT200, CT1500 and CT600. The front panel of the associated precise measuring resistor box is presented in Fig. 7 (below).

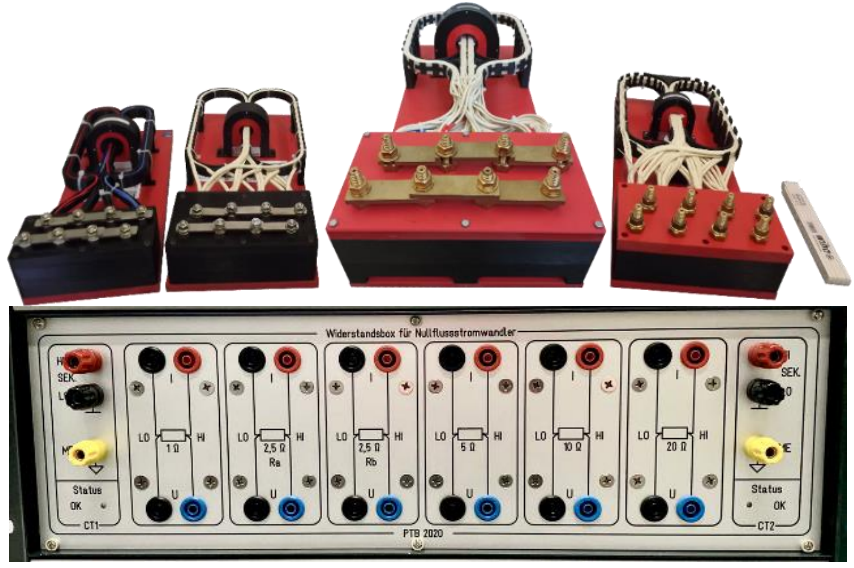

Fig. 7. Photo of the accomplished wideband current-to-voltage transformer set for currents up to $2 \mathrm{kA}$. The symmetrical current transformers (above) and the front panel of the associated precise measuring resistor box (below)

According to the previous work [8], the characterisation of the C-to-V transformers was carried out by separating the behaviour at power frequency and the frequency response. The errors of the current transformers $\left(\varepsilon_{\mathrm{i}}(f)\right.$ and $\left.\delta_{\mathrm{i}}(f)\right)$ are represented by $\varepsilon_{\mathrm{i}}(f)=\varepsilon_{\mathrm{i}}\left(f_{0}\right)+\Delta \varepsilon_{\mathrm{i}}(f)$ and $\delta_{\mathrm{i}}(f)=\delta_{\mathrm{i}}\left(f_{0}\right)+\Delta \delta_{\mathrm{i}}(f)$, where $\varepsilon_{\mathrm{i}}\left(f_{0}\right)$ and $\delta_{\mathrm{i}}\left(f_{0}\right)$ refer to the $\mathrm{CT}$ errors at power frequency and $\Delta \varepsilon_{\mathrm{i}}(f)$ and $\Delta \delta_{\mathrm{i}}(f)$ refer to the frequency response of the CTs. A step-up calibration was especially used when the frequency response of the CTs is determined. So far, the calibration measurements at power frequency of the reference current transformer set have been completed. The errors of the reference current transformer set were within $\pm 10 \mu \mathrm{A} / \mathrm{A}$ and $\mu \mathrm{rad}$ at $50 \mathrm{~Hz}$. In addition, the initial frequency response measurements of the first two CTs (CT50 and CT200) showed that the ac errors at a frequency of $12 \mathrm{kHz}$ were below $0.1 \%$ and $0.2 \mathrm{crad}$ with the expanded uncertainties are below $0.01 \%$ and $0.03 \mathrm{crad}(k=2)$.

Furthermore, the errors of the self-developed measuring resistors are represented by a ratio error $\varepsilon_{\mathrm{R}}$ and a time constant $\tau_{\mathrm{Z}}$, where $\varepsilon_{\mathrm{R}}$ was obtained by the calibration at power frequency and $\tau_{\mathrm{Z}}$ was derived from the phase behaviour of the frequency response measurements. As a consequence, the calibrated results of the 6 measuring resistors are listed in TABLE II.

TABLE II. ERRORS OF MEASURING RESISTORS. RATIO ERRORS OF RESISTORS AT POWER FREQUENCY WITH 100\% RATED INPUT CURRENT AND PHASE ERRORS OF RESISTORS FOR FREQUENCIES FROM 16 HZ TO 12 KHZ WITH $80 \%$ RATED INPUT CURRENT

\begin{tabular}{|c|c|c|c|c|c|}
\hline $\boldsymbol{R}_{\mathrm{m}}$ & $\boldsymbol{I}_{\text {nom }}$ & $\begin{array}{c}\boldsymbol{\varepsilon}_{\mathrm{R}} \text { in } \\
\boldsymbol{\mu} \boldsymbol{\Omega} / \boldsymbol{\Omega}\end{array}$ & $\begin{array}{c}\boldsymbol{U}_{\mathbf{\varepsilon}} \\
\text { in } \boldsymbol{\mu} \boldsymbol{\Omega} / \boldsymbol{\Omega} \\
(\boldsymbol{k}=\mathbf{2})\end{array}$ & $\begin{array}{c}\boldsymbol{\tau}_{\mathrm{Z}} \\
\text { in } \mathbf{~ n s}\end{array}$ & $\begin{array}{c}\boldsymbol{U}\left(\boldsymbol{\tau}_{\mathrm{Z}}\right) \\
\text { in ns } \\
(\boldsymbol{k}=\mathbf{2})\end{array}$ \\
\hline $\mathbf{1} \boldsymbol{\Omega}$ & $\mathbf{1} \mathbf{A}$ & -1.2 & 2.5 & 0.2 & 4 \\
\hline $\mathbf{2 . 5} \boldsymbol{\Omega}-\boldsymbol{R}_{\mathbf{a}}$ & $\mathbf{4 0 0} \mathbf{~} \mathbf{A}$ & 0.7 & 2.4 & 0.0 & 3 \\
\hline $\mathbf{2 . 5} \boldsymbol{\Omega}-\boldsymbol{R}_{\mathbf{b}}$ & $\mathbf{4 0 0} \mathbf{~} \mathbf{A}$ & 0.3 & 2.4 & 0.0 & 3 \\
\hline $\mathbf{5} \boldsymbol{\Omega}$ & $\mathbf{2 0 0} \mathbf{~} \mathbf{A}$ & 0.6 & 2.5 & 0.0 & 3 \\
\hline $\mathbf{1 0} \boldsymbol{\Omega}$ & $\mathbf{1 0 0} \mathbf{~} \mathbf{A}$ & -1.6 & 1.7 & -0.3 & 3 \\
\hline $\mathbf{2 0} \boldsymbol{\Omega}$ & $\mathbf{5 0} \mathbf{~} \mathbf{A}$ & -2.0 & 2.5 & -1.1 & 3 \\
\hline
\end{tabular}

The ratio errors of the 6 measuring resistors were below $\pm 2 \mu \Omega / \Omega$ at power frequency with expanded uncertainties below $\pm 3 \cdot 10^{-6}(k=2)$. The time constant was about below $\pm 1 \mathrm{~ns}$ with the attained expanded uncertainties below $\pm 4 \mathrm{~ns}$.

\section{Validation of the SVs}

To validate the accuracy of the received SVs from the SV receiver box, the plausibility of the generated SVs needs to be validated in the first place. Therefore, the validation measurements the SVs are assigned into two parts: the validation of the SV generation and the validation of the SV receiving.

For laboratory validation of the SV generation, a microcontroller-based SV generator box and the corresponding LabView-based program were developed for sending the SV-data over the ethernet using the IEC91850-92 communication protocol. The basic module box is a 32-bit ARM Cortex-M4 CPU with an ethernet and a USB port. Three-phase four-wire $\left(L_{1}, L_{2}, L_{3}, L_{\mathrm{N}}\right)$ currents and voltages with $1 \mathrm{~mA}$ current resolution and $10 \mathrm{mV}$ voltage resolution are programmed as the SV data stream though the USB port. A one-period signal is initially programmed to the SV generator box and repetitively sent to the SV-based devices. The beginning of an SV data set is signalized by a rising edge on the PPS output. The sample rates for the SV generation were configured as $4000 \mathrm{~Hz}$ (Application Service Data Unit: ASDU $=1), 4800 \mathrm{~Hz}(\mathrm{ASDU}=1$ or $\mathrm{ASDU}=2), 5760 \mathrm{~Hz}$ $(\mathrm{ASDU}=1)$ and $14400 \mathrm{~Hz}(\mathrm{ASDU}=6)$. In addition, the SV generator box was considered as a substitute for the devices with the digital output (e.g., a SAMU) and can be used for the validation of an SV-based measuring device (e.g., for digital energy meters) without any numerical loss.

The validation of the SV generation mainly deals with the round-off function in LabVIEW program. The errors were determined by the currents and voltages difference between the SVs directly generated by LabVIEW and the SVs sent to the SV-based devices. The results of the SV generation for $L_{1}$ 
are presented in Fig. 8 for instance. Dual-tone signals $\left(f_{0}=50 \mathrm{~Hz}, I_{0}=1 \mathrm{kA}, \varphi_{0 \_\mathrm{I}}=0^{\circ}, U_{0}=100 \mathrm{kV}, \varphi_{0 \_\mathrm{U}}=0^{\circ}\right)$ with the $3^{\text {rd }}$ harmonics $(10 \%$ amplitude, zero phase offset) were generated for the three-phase currents and voltages.

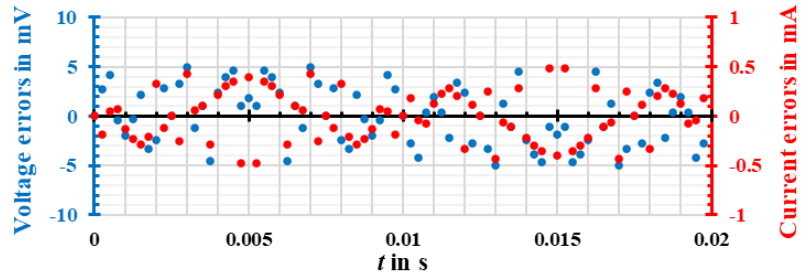

Fig. 8. The errors of generated voltages and currents $\left(L_{1}\right)$ in SV generator program

As shown in Fig. 8, the voltage errors stay within $\pm 5 \mathrm{mV}$ and the current errors stay within $\pm 0.5 \mathrm{~mA}$, as anticipated due to the $1 \mathrm{~mA}$ current resolution and $10 \mathrm{mV}$ voltage resolution of the standardised data stream [1], [2]. It can therefore be concluded that the sampled values, sent by the SV generator box, are plausible and numerically accurate within their resolutions.

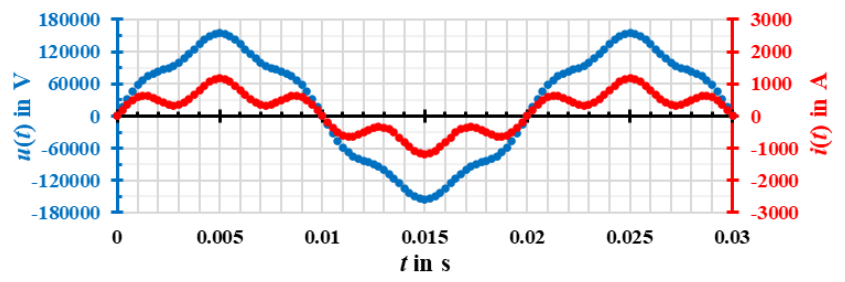

Fig. 9. The received voltages and currents $\left(L_{1}\right)$ of SV receiver box in time domain

Corresponding to the SV generation for Fig. 8, the received $\mathrm{SV}$ s of $L_{1}$ from the $\mathrm{SV}$ receiver box are shown in Fig. 9. The diagram shows that the SV receiver box received the repeated one-period signal ( $t>0.02 \mathrm{~s}$ ) from the SV generator box. Moreover, the differences between the sent and received $\mathrm{SVs}$ equalled to zero. This means that the SV receiver box receives exactly what is sent to it. Eventually, similar results were obtained by $L_{2}, L_{3}$ and $L_{\mathrm{N}}$ as well.

\section{CONCLUSION}

In conclusion, the proposed calibration system has been applied for calibrating at least three different types of analogue current sensors (conventional inductive CTs, electronic CTs and Rogowski coils) and two SAMUs from different companies, as planned in FutureGrid II. The absolute phase errors of the two-channel generator to the GPS PPS time reference are mainly depended on the $\mathrm{SpS}$ and can be configured to almost zero. The errors of the reference current transformer set are within $\pm 10 \mu \mathrm{A} / \mathrm{A}$ and $\mu \mathrm{rad}$ at power frequency. According to IEC 61869-13 [5] for a SAMU and IEC 61869-6 [16] for a low-power instrument transformer (LPIT), the anti-aliasing filter attenuation for the half of the sampling rates (up to $14.4 \mathrm{kHz}$ ) up to $7.2 \mathrm{kHz}$ shall be greater and equal to $40 \mathrm{~dB}$. The uncertainties caused by the twochannel generator, the set of reference current transformers and the precision 2-channel measuring system are well below $10^{-3}$ up to $12 \mathrm{kHz}$. Compared to the limits by the internal antialiasing filter of the DUT, the uncertainties of the proposed measuring system strictly meet the requirements. The SV receiver box is firstly validated for all sample rates with 1 ASDU. Additionally, preliminary calibrations of a SAMU are currently being executed.
Further complete calibrations of the integrated C-to- $\mathrm{V}$ transformer set and the SAMU as well as the corresponding measurement uncertainties will be accomplished in the prospective work. The SV receiver box will be extended for further sample rates with 2 ASDUs and 6 ASDUs according to [1]. Moreover, small phase errors of the phase reference signal of the generator can be software-compensated with a non-standard phase correction.

\section{ACKNOWLEDGMENT}

This project has received funding from the EMPIR programme co-financed by the Participating States and from the European Union's Horizon 2020 research and innovation programme.

\section{REFERENCES}

[1] Instrument Transformers - Part 9: Digital interface for instrument transformers, IEC 61869-9, 2016.

[2] Communication networks and systems for power utility automation Part 9-2: Specific communication service mapping (SCSM) - Sampled values over ISO/IEC 8802-3, IEC 61850-9-2, 2005.

[3] (2017) The Future Grid II website [Online]. Available: https://www.vtt.fi/sites/FutureGrid2/ [May 2021].

[4] T. A. Lehtonen, "Harmonic Power Standard of VTT MIKES," in IEEE Transactions on Instrumentation and Measurement, vol. 68, no. 6, pp. 2047-2052, June 2019, doi: 10.1109/TIM.2018.2880056.

[5] Instrument Transformers - Part 13: Stand-alone merging unit (SAMU), IEC 61869-13, 2019.

[6] B. Djokic and H. Parks, "A Synchronized Current-Comparator-Based Power Bridge for Calibrating Analog Merging Units," 2018 Conference on Precision Electromagnetic Measurements (CPEM 2018), 2018, pp. 1-2, doi: 10.1109/CPEM.2018.8501127.

[7] B. Djokic and H. Parks, "Calibration of Electricity Meters with Digital Input," 2018 Conference on Precision Electromagnetic Measurements (CPEM 2018), 2018, pp. 1-2, doi: 10.1109/CPEM.2018.8500803.

[8] Chen Y., Mohns E., Badura H., Raether P., " Development of a Wideband Current-to-Voltage Transformer Set for Currents up to 2 kA", 2020 Conference on precision Electromagnetic measurements (CPEM), 24-28 August 2020; doi: 10.1109/CPEM49742.2020.9191814.

[9] E. Mohns, J. Meisner, G. Roeissle, M. Seckelmann and M. Korejwo: "A Wideband Current Transformer Bridge," 4rd IEEE International Workshop on Applied Measurements for Power Systems, AMPS 2013, September 2013, Aachen, Germany.

[10] T.Möhring, E.Mohns, M.Schmidt, T.Funck: "Characterization of a 2Channel Digitizer with Differential Inputs," CPEM 2012 Digest, Washington, July 2012.

[11] White Rabbit LEN [Online]. Available: https://sevensols.com/home/timing-products/wr-len/ [May 2021].

[12] E. Mohns, A. Mortara, H. Cayci, E. Houtzager, S. Fricke, M. Agustoni, B. Ayhan: "Calibration of Commercial Test Sets for Non-Conventional Instrument Transformers," IEEE International Workshop on Applied Measurements for Power Systems, AMPS 2017, September 2017, Liverpool, UK, DOI: 10.1109/AMPS.2017.8078324.

[13] H.-D. Schlemper, D. Füchsle, G. Ramm, J. Widmer: "Test and Application of Non-Conventional Multi-Purpose Voltage and Current Transducers", CIGRE, Session 2004, A3-108.

[14] Y. Chen, E. Mohns, M. Seckelmann and d.R. Soeren, "Precise Amplitude and Phase Determination Using Resampling Algorithms for Calibrating Sampled Value Instruments," Sensors. 2020; 20(24):7345. pp. 1-21, doi: https://doi.org/10.3390/s20247345.

[15] E. Mohns, J. Chunyang, H. Badura and P. Raether: "A Fundamental Step-Up Method for Standard Voltage Transformers Based on an Active Capacitive High-Voltage Divider," IEEE Trans. Instrum. Meas., vol. 68, no. 6, pp. 2121-2128, June 2019, DOI: 10.1109/TIM.2018.2880055.

[16] Instrument Transformers - Part 6: Additional general requirements for low-power instrument transformers, IEC 61869-6, 2016. 